\title{
Two Randomized Trials of Canakinumab in Systemic Juvenile Idiopathic Arthritis
}

\author{
Nicolino Ruperto, M.D., M.P.H., Hermine I. Brunner, M.D., Pierre Quartier, M.D., \\ Tamás Constantin, M.D., Nico Wulffraat, M.D., Gerd Horneff, M.D., Riva Brik, M.D., \\ Liza McCann, M.D., Ozgur Kasapcopur, M.D., Lidia Rutkowska-Sak, M.D., \\ Rayfel Schneider, M.D., Yackov Berkun, M.D., Inmaculada Calvo, M.D., \\ Muferet Erguven, M.D., Laurence Goffin, M.D., Michael Hofer, M.D., \\ Tilmann Kallinich, M.D., Sheila K. Oliveira, M.D., Yosef Uziel, M.D., Stefania Viola, M.D., \\ Kiran Nistala, M.D., Carine Wouters, M.D., Rolando Cimaz, M.D., Manuel A. Ferrandiz, M.D., \\ Berit Flato, M.D., Maria Luz Gamir, M.D., Isabelle Kone-Paut, M.D., Alexei Grom, M.D., \\ Bo Magnusson, M.D., Seza Ozen, M.D., Flavio Sztajnbok, M.D., Karine Lheritier, Ph.D., \\ Ken Abrams, M.D., Dennis Kim, M.D., M.P.H., Alberto Martini, M.D., \\ and Daniel J. Lovell, M.D., M.P.H., for the PRINTO and PRCSG*
}

The authors' affiliations are listed in the Appendix. Address reprint requests to Dr. Ruperto at Istituto Giannina Gaslini, PRINTO, Via Gaslini 5, 16147 Genoa, Italy, or at nicolaruperto@ospedale-gaslini.ge.it.

Drs. Ruperto, Brunner, Martini, and Lovell contributed equally to the article.

*Additional investigators for the Paediatric Rheumatology International Trials Organisation (PRINTO) and the Pediatric Rheumatology Collaborative Study Group (PRCSG) are listed in the Supplementary Appendix, available at NEJM.org

N Engl J Med 2012;367:2396-406. DOI: $10.1056 /$ NEJMoal205099

Copyright (c) 2012 Massachusetts Medical Society.

\section{A B STRACT}

\section{BACKGROUND}

Interleukin-1 is pivotal in the pathogenesis of systemic juvenile idiopathic arthritis (JIA). We assessed the efficacy and safety of canakinumab, a selective, fully human, anti-interleukin- $1 \beta$ monoclonal antibody, in two trials.

METHODS

In trial 1 , we randomly assigned patients, 2 to 19 years of age, with systemic JIA and active systemic features (fever; $\geq 2$ active joints; C-reactive protein, $>30 \mathrm{mg}$ per liter; and glucocorticoid dose, $\leq 1.0 \mathrm{mg}$ per kilogram of body weight per day), in a double-blind fashion, to a single subcutaneous dose of canakinumab (4 mg per kilogram) or placebo. The primary outcome, termed adapted JIA ACR 30 response, was defined as improvement of $30 \%$ or more in at least three of the six core criteria for JIA, worsening of more than $30 \%$ in no more than one of the criteria, and resolution of fever. In trial 2, after 32 weeks of open-label treatment with canakinumab, patients who had a response and underwent glucocorticoid tapering were randomly assigned to continued treatment with canakinumab or to placebo. The primary outcome was time to flare of systemic JIA.

\section{RESULTS}

At day 15 in trial 1, more patients in the canakinumab group had an adapted JIA ACR 30 response (36 of 43 [84\%], vs. 4 of 41 [10\%] in the placebo group; $\mathrm{P}<0.001$ ). In trial 2, among the 100 patients (of 177 in the open-label phase) who underwent randomization in the withdrawal phase, the risk of flare was lower among patients who continued to receive canakinumab than among those who were switched to placebo ( $74 \%$ of patients in the canakinumab group had no flare, vs. $25 \%$ in the placebo group, according to Kaplan-Meier estimates; hazard ratio, 0.36; $\mathrm{P}=0.003$ ). The average glucocorticoid dose was reduced from 0.34 to $0.05 \mathrm{mg}$ per kilogram per day, and glucocorticoids were discontinued in 42 of 128 patients (33\%). The macrophage activation syndrome occurred in 7 patients; infections were more frequent with canakinumab than with placebo.

\section{CONCLUSIONS}

These two phase 3 studies show the efficacy of canakinumab in systemic JIA with active systemic features. (Funded by Novartis Pharma; ClinicalTrials.gov numbers, NCT00889863 and NCT00886769.) 
YSTEMIC JUVENILE IDIOPATHIC ARTHRItis (JIA), the most severe JIA subtype, is characterized by chronic arthritis; intermittently high, spiking temperatures; maculopapular rash; hepatosplenomegaly; lymphadenopathy; serositis; and a marked increase in the level of acute-phase reactants. ${ }^{1-3}$ Complications of systemic JIA include growth impairment, osteoporosis, and the potentially lethal macrophage activation syndrome. ${ }^{4-6}$

Until recently, systemic JIA was considered a therapeutic orphan, since the principal effective treatment was glucocorticoids, with their known toxicity and long-term growth and bone sequelae. ${ }^{7}$ Other therapeutic options include nonsteroidal antiinflammatory drugs (NSAIDs), methotrexate, and biologic agents. Both interleukin- $6^{8-10}$ and, more recently, interleukin-111-14 have been found to be central to the pathogenesis of systemic JIA. However, reported evidence of the benefits of anti-interleukin-1 treatment remains limited.

Canakinumab is a fully human, anti-interleukin- $1 \beta$ monoclonal antibody that selectively binds to interleukin- $\beta$, inactivating its signaling. ${ }^{15-17}$ Data from a recent phase 2 trial led to the dose of $4 \mathrm{mg}$ per kilogram of body weight per month (maximum dose, $300 \mathrm{mg}$ ), administered subcutaneously, that was used in this phase 3 trial. ${ }^{18} \mathrm{We}$ assessed the efficacy and safety of canakinumab for the treatment of systemic JIA with active systemic features in two phase 3, randomized, double-blind, placebocontrolled studies.

\section{METHODS}

\section{PATIENTS}

Eligible patients were 2 to 19 years of age, with systemic JIA, ${ }^{19,20}$ including active systemic features and arthritis (intermittently spiking temperatures of $>38^{\circ} \mathrm{C}, \geq 2$ active joints, ${ }^{21}$ and C-reactive protein level of $>30 \mathrm{mg}$ per liter [normal value, $<10 \mathrm{mg}$ per liter]). Background therapy with a prednisone equivalent of up to $1.0 \mathrm{mg}$ per kilogram per day and stable doses of NSAIDs and methotrexate $(\leq 20 \mathrm{mg}$ per square meter of body-surface area per week) were permitted. Major exclusion criteria were concomitant treatment with another biologic agent or disease-modifying drug (with a washout of $\geq 5$ half-lives), diagnosis of the macrophage activation syndrome 6 within 6 months before enrollment, active tuberculosis, and live-virus vaccination within 3 months before enrollment.

\section{STUDY DESIGN}

Patients were enrolled in the two trials between July 2009 and December 2010 at 63 centers that were members of the Paediatric Rheumatology International Trials Organisation (PRINTO) ${ }^{22}$ and the Pediatric Rheumatology Collaborative Study Group (PRCSG). Trial 1 was a 29-day, single-dose, randomized, double-blind, placebo-controlled study. Patients with persistent fever (temperature, $>38^{\circ} \mathrm{C}$ ) after day 3 were made aware of the study assignment at the physician's discretion and, if they were receiving placebo, were permitted to enroll in trial 2. In trial 1, patients who had a response to canakinumab at day 15 , according to an adaptation of the JIA American College of Rheumatology (ACR) 30 response (adapted JIA ACR 30 response, defined as the absence of fever plus improvement of $\geq 30 \%$ in three or more of the six variables of the JIA core set, ${ }^{22}$ with no more than one variable worsening by $>30 \%$ at day $15^{23,24}$ ), were immediately enrolled in trial 2 on day 29 (Fig. S1 and S2 in the Supplementary Appendix, available with the full text of this article at NEJM.org).

Trial 2 used a two-part withdrawal design. ${ }^{24,25}$ The first part was an open-label phase in which eligible patients were treated with canakinumab every 4 weeks for 12 to 32 weeks. For patients who were receiving glucocorticoids at enrollment, glucocorticoid tapering according to a standardized procedure was permitted from week 9 through week 28, if there was at least an adapted JIA ACR 50 response (indicating the absence of fever and an improvement of $\geq 50 \%$ in at least three of the six core criteria for JIA, with a worsening of $>30 \%$ in no more than one of the criteria). The second part was a withdrawal phase in which patients who had at least an adapted JIA ACR 30 response that was sustained and who were not receiving glucocorticoids or who had undergone successful glucocorticoid tapering and were receiving a stable dose of glucocorticoids were randomly assigned in a 1:1 ratio, in a doubleblind fashion, to continued treatment with canakinumab or to placebo (for glucocorticoidtapering recommendations and eligibility criteria for randomization, see Table S1 in the Supplementary Appendix). In this withdrawal phase, patients who had a disease flare according to a prespecified definition were treated again with canakinumab in an open-label fashion. All patients who did not undergo glucocorticoid tapering, who had no response during the open-label 
phase, or who had a flare during the withdrawal phase could enter the ongoing long-term, openlabel extension phase of the trial. In both trials, randomization was computer-generated by means of an interactive voice-response system, with a block size of two.

\section{STUDY OVERSIGHT}

The protocols of the two trials were approved by the local ethics committees, and written informed consent and assent were obtained. The trials were conducted in accordance with Good Clinical Practice guidelines and the study protocols, available at NEJM.org.

The studies were designed jointly by four of the academic authors and the sponsor (Novartis Pharma), with data collected by the PRINTO and PRCSG investigators. The sponsor was responsible for the overall management of the study and the data analysis.

The initial draft of the manuscript was written by the first author, with critical revision by three of the academic authors; these four authors decided to submit the manuscript for publication. All authors contributed to the revision. All the academic authors vouch for the completeness and veracity of the data and analyses, as well as for the fidelity of the study to the protocol. Editorial assistance was provided by a medical writer paid by the sponsor.

\section{ASSESSMENT AND OUTCOMES}

Clinical assessments were performed at days 3, 15, and 29 and every 28 days thereafter. The primary outcome for trial 1 was the proportion of patients with an adapted JIA ACR 30 response. Resolution of fever was defined as the documented absence of fever due to systemic JIA (body temperature, $\leq 38^{\circ} \mathrm{C}$ ) in the week preceding the assessment, according to a paper diary. The six JIA core-set variables (with higher values indicating higher disease activity) were the number of joints with active arthritis ( 0 to 73 joints), ${ }^{21}$ the number of joints with limited range of motion ( 0 to 69 joints), the physician's global assessment of disease activity (on a $100-\mathrm{mm}$ visual-analogue scale, with higher scores indicating more disease activity), the parent's global assessment of the patient's overall well-being (on a 100-mm visual-analogue scale, with higher scores indicating worse overall wellbeing), an assessment of physical function with the use of the cross-culturally adapted and vali- dated version of the Disability Index of the Childhood Health Assessment Questionnaire (CHAQ-DI; on a scale of 0 to 3 , with higher scores indicating greater disability), and a C-reactive protein level standardized to 0 to $10 \mathrm{mg}$ per liter..$^{24,26-28} \mathrm{An}$ independent joint examiner was required at each center; 41 of the 63 centers ( $65 \%$ ) had at least one joint examiner who was certified by PRINTO or PRCSG.

In the open-label phase of trial 2, the objective was to determine whether at least $25 \%$ of the patients who were being treated with glucocorticoids could have their dose tapered (Table S1 in the Supplementary Appendix); the expected tapering frequency was established by consensus of the steering committee. In the withdrawal phase, the objective was to show that the time to flare was longer with canakinumab than with placebo. Flare was defined as the recurrence of fever lasting for 2 or more consecutive days; a worsening of $30 \%$ or more in three or more of the six variables of the JIA core set, with no more than one variable improving by $30 \%$ or more (minimum contingencies included worsening in at least two joints with active or limited disease, at least a 20-mm worsening on the visual-analogue scale in the physician's or parent's global assessment of disease activity, and a C-reactive protein level of $30 \mathrm{mg}$ per liter) ${ }^{18,23,29}$; or discontinuation of treatment, except in the case of discontinuation because of inactive disease at 24 weeks or more.

Patients were also evaluated for higher levels of improvement, including adapted JIA ACR 50, JIA ACR 70, JIA ACR 90, and JIA ACR 100 responses (defined as absence of fever plus improvements of $\geq 50 \%, \geq 70 \%, \geq 90 \%$, and $100 \%$, respectively, in at least three of the six response variables and a worsening of $>30 \%$ in no more than one of the six variables), and inactive disease (i.e., absence of active arthritis, fever, and signs or symptoms of systemic JIA; normal C-reactive protein level; and physician's global assessment of disease activity of $\leq 10 \mathrm{~mm}$ ). ${ }^{23,30-33}$ Safety assessments included the collection of data on adverse events and serious adverse events. The primary outcome evaluation and assessments of flare, inactive disease, and glucocorticoid-tapering recommendations were performed in real time, according to validated criteria, ${ }^{23,24,29,30}$ by independent evaluators at the PRINTO and PRCSG coordinating centers who were unaware of the study assignments. 
STATISTICAL ANALYSIS

The trials followed the recommendation of the Consolidated Standards of Reporting Trials (CONSORT) statement, ${ }^{34}$ with results reported for the intention-to-treat population. Baseline characteristics were summarized with the use of descriptive statistics. The adapted JIA ACR level of improvement was calculated with reference to the day of the first injection of canakinumab, whereas flare was assessed with respect to the core-set variables on the day of randomization in the withdrawal phase of trial 2. Patients who withdrew from trial 2 were considered not to have a response from date of withdrawal onward.

For trial 1, we calculated that a sample of 122 patients would be required for the trial to have $90 \%$ power to detect a $30 \%$ difference in the proportions of patients with an adapted JIA ACR 30 response, at a one-sided significance level of 0.025 . The trial was terminated early on the recommendation of the independent data and safety monitoring committee on the basis of one interim analysis (according to a priori rules in the protocol amendment), showing that the primary end point had been achieved at a significance level of 0.00697 . For trial 2 , to achieve $90 \%$ power, a total of 37 events (flares) were needed in the withdrawal phase (13 events in the canakinumab group and 24 in the placebo group). We estimated that 214 patients would need to be enrolled in the open-label phase in order to randomly assign 58 patients to study groups in the withdrawal phase.

\section{RESULTS}

\section{STUDY POPULATION}

Table 1 summarizes the baseline disease characteristics for a total of 190 unique participants. Baseline characteristics were balanced between the two treatment groups in trial 1 and in trial 2, according to the randomization in the withdrawal phase. More than two thirds of the patients had received biologic agents previously. Figure S2 in the Supplementary Appendix shows the study-group assignments in the two trials. In the open-label phase of trial 2, a total of 77 of 177 patients (44\%) discontinued the study or entered the ongoing open-label extension phase; the most frequently reported reasons were lack of response to therapy by day 29 (in 34 patients) or an inability to undergo glucocorticoid tapering (in 24). A total of 144 of 177 patients (81\%) entered the ongoing, long-term, open-label extension study.

\section{EFFICACY}

At day 15 of trial 1, a total of 36 patients in the canakinumab group (84\%), as compared with 4 in the placebo group (10\%), had an adapted JIA ACR 30 response, which was sustained at day 29 $(\mathrm{P}<0.001)$ (Fig. 1A). At the end of the open-label phase of trial 2, after a median of 113 days and a median of four injections of canakinumab, 128 of 175 patients $(73 \%)$ had at least an adapted JIA ACR 50 response, and 55 of 176 (31\%) had inactive disease. A total of 100 of 177 patients (56\%) underwent glucocorticoid tapering, had at least an adapted JIA ACR 30 response, and were eligible to undergo randomization in the withdrawal phase of trial 2. Denominators differ because assessments were missing for some patients at some end points.

In the withdrawal phase, the median time to flare was 236 days ( $95 \%$ confidence interval [CI], 141 to 449) in the placebo group; the median was not observable in the canakinumab group, since less than $50 \%$ of the patients had a flare $(\mathrm{P}=0.003$ by the log-rank test) (Fig. $\mathrm{S} 3$ in the Supplementary Appendix). A total of 39 patients in the canakinumab group ( $74 \%$, according to the Kaplan-Meier estimate) had no flare, as compared with 24 in the placebo group (25\%, according to the Kaplan-Meier estimate), with a significant relative risk reduction of $64 \%$ with regard to flare. At the end of the withdrawal phase, a total of 31 of 50 patients in the canakinumab group (62\%) had inactive disease, as compared with 17 of 50 in the placebo group (34\%) (Fig. 1B). Table 2 shows the changes over time in the JIA core-set measures and clinical features for the patients in the two trials (see Table S2 in the Supplementary Appendix for a list of laboratory tests).

\section{GLUCOCORTICOID TAPERING}

Glucocorticoids were prescribed for 128 of the 177 patients (72\%) at study entry, with 57 of these 128 patients (45\%; 90\% CI, 37 to 52; $\mathrm{P}<0.001)$ able to undergo glucocorticoid tapering from a mean dose of $0.34 \mathrm{mg}$ per kilogram per day to $0.05 \mathrm{mg}$ per kilogram per day. A total of 42 of the 128 patients (33\%) discontinued glucocorticoids completely. 


\begin{tabular}{|c|c|c|c|c|c|}
\hline \multirow[t]{2}{*}{ Characteristic } & \multicolumn{2}{|c|}{ Trial 1} & \multirow{2}{*}{$\begin{array}{c}\text { Trial 2, } \\
\text { Open-Label Phase } \\
\text { Canakinumab } \\
(\mathrm{N}=177)\end{array}$} & \multicolumn{2}{|c|}{$\begin{array}{c}\text { Trial 2, } \\
\text { Withdrawal Phase }\end{array}$} \\
\hline & $\begin{array}{l}\text { Canakinumab } \\
\quad(\mathrm{N}=43)\end{array}$ & $\begin{array}{l}\text { Placebo } \\
(\mathrm{N}=41)\end{array}$ & & $\begin{array}{l}\text { Canakinumab } \\
\qquad(\mathrm{N}=50)\end{array}$ & $\begin{array}{l}\text { Placebo } \\
(\mathrm{N}=50)\end{array}$ \\
\hline \multicolumn{6}{|l|}{ Sex - no. (\%) } \\
\hline Male & $16(37)$ & $18(44)$ & $79(45)$ & $22(44)$ & $23(46)$ \\
\hline Female & $27(63)$ & $23(56)$ & $98(55)$ & $28(56)$ & $27(54)$ \\
\hline \multicolumn{6}{|l|}{ Race - no. (\%)† } \\
\hline White & $40(93)$ & $37(90)$ & $151(85)$ & $41(82)$ & $42(84)$ \\
\hline Black & $2(5)$ & 0 & $7(4)$ & $2(4)$ & $1(2)$ \\
\hline Other & $1(2)$ & $4(10)$ & $19(11)$ & $7(14)$ & $7(14)$ \\
\hline \multicolumn{6}{|l|}{ Age $-y r$} \\
\hline Median & 8.0 & 9.0 & 8.0 & 8.0 & 8.0 \\
\hline Interquartile range & $4.0-13.0$ & $6.0-14.0$ & $5.0-12.0$ & $6.0-12.0$ & $5.0-13.0$ \\
\hline \multicolumn{6}{|l|}{ Body weight - kg } \\
\hline Median & 22.2 & 27.2 & 25.8 & 28.6 & 25.9 \\
\hline Interquartile range & $15.1-47.9$ & $21.0-45.5$ & $17.8-42.9$ & $19.8-44.0$ & $18.6-49.4$ \\
\hline \multicolumn{6}{|l|}{ Disease duration $-\mathrm{yr}$} \\
\hline Median & 2.3 & 2.0 & 2.1 & 2.7 & 1.8 \\
\hline Interquartile range & $1.0-4.7$ & $1.2-5.2$ & $0.8-4.3$ & $1.3-6.2$ & $0.4-4.3$ \\
\hline Use of methotrexate at baseline - no. (\%) & $29(67)$ & $24(59)$ & $93(53)$ & $28(56)$ & $26(52)$ \\
\hline Prior use of biologic agent - no. (\%) $\ddagger$ & $25(58)$ & $23(56)$ & $116(66)$ & $30(60)$ & $27(54)$ \\
\hline Anakinra & $16(37)$ & $15(37)$ & $83(47)$ & $25(50)$ & $20(40)$ \\
\hline Tocilizumab & $1(2)$ & $2(5)$ & $10(6)$ & $4(8)$ & $1(2)$ \\
\hline Anti-TNF agent or other biologic agent & $14(33)$ & $16(39)$ & $62(35)$ & $14(28)$ & $12(24)$ \\
\hline Prednisone therapy at baseline - no. (\%) & $31(72)$ & $28(68)$ & $128(72)$ & $32(64)$ & $30(60)$ \\
\hline
\end{tabular}

* There were no significant differences between the two treatment groups in trial 1 and in trial 2, according to the randomization in the withdrawal phase.

$\uparrow$ Race was determined by the investigators.

$\downarrow$ A patient could have received one or more biologic agents previously. TNF denotes tumor necrosis factor.

\section{SAFETY}

Table 3 summarizes adverse events that occurred during the two trials. No patient in trial 1 discontinued the study because of an adverse event; in the withdrawal phase of trial 2, three patients in the placebo group withdrew because of a serious adverse event (the macrophage activation syndrome, pneumonia, and flare of systemic JIA). Another three patients in the placebo group withdrew because of nonserious adverse events (vomiting, rash, and uveitis).

In trial 1, two serious adverse events were reported in each group. In the canakinumab group, there was one episode each of the macrophage activation syndrome and varicella; in the placebo group, there was one episode each of the macrophage activation syndrome and gastroenteritis.

In the open-label phase of trial 2, seven patients had serious nonopportunistic infections: two were associated with the macrophage activation syndrome, and five resolved within 2 weeks.

In the withdrawal phase, serious adverse events occurred in six patients in the canakinumab group (arm pain and lymphadenopathy in one; otitis media and leg fracture in one; leukopenia, thrombocytopenia, and aminotransferase elevations in one; aminotransferase elevations in one; respiratory tract infection in one; and splenic cyst in one); none of these events led to discontinuation of the study drug. Serious adverse events occurred in six patients who received placebo (the macrophage 
activation syndrome and urosepsis in one, measles and pneumonia in one, traumatic fracture in two, and flare of systemic JIA in two); three of these patients (one of the two patients with a disease flare, the patient with the macrophage activation syndrome and urosepsis, and the patient with measles and pneumonia) discontinued the study drug because of the event. There were no cases of cancer, tuberculosis, or opportunistic infection in either trial. Anti-canakinumab antibodies were detected in four patients; none were neutralizing.
In trial 1, thrombocytopenia developed in 2 patients in the canakinumab group (5\%) and in 1 of 38 patients in the placebo group (3\%), and neutropenia developed in 2 patients in the canakinumab group (5\%) and none in the placebo group. In the open-label phase of trial 2, a total of 11 of 176 patients with assessments (6\%) had thrombocytopenia, and 10 of 176 (6\%) had neutropenia. In the withdrawal phase of trial 2, a total of 3 patients in the canakinumab group $(6 \%)$ and 1 in the placebo group $(2 \%)$ had

Adapted JIA ACR $30 \quad \square$ Adapted JIA ACR $50 \quad \square$ Adapted JIA ACR $70 \quad \square$ Adapted JIA ACR $90 \quad \square$ Adapted JIA ACR $100 \quad \square$ Inactive disease

A Trial 1
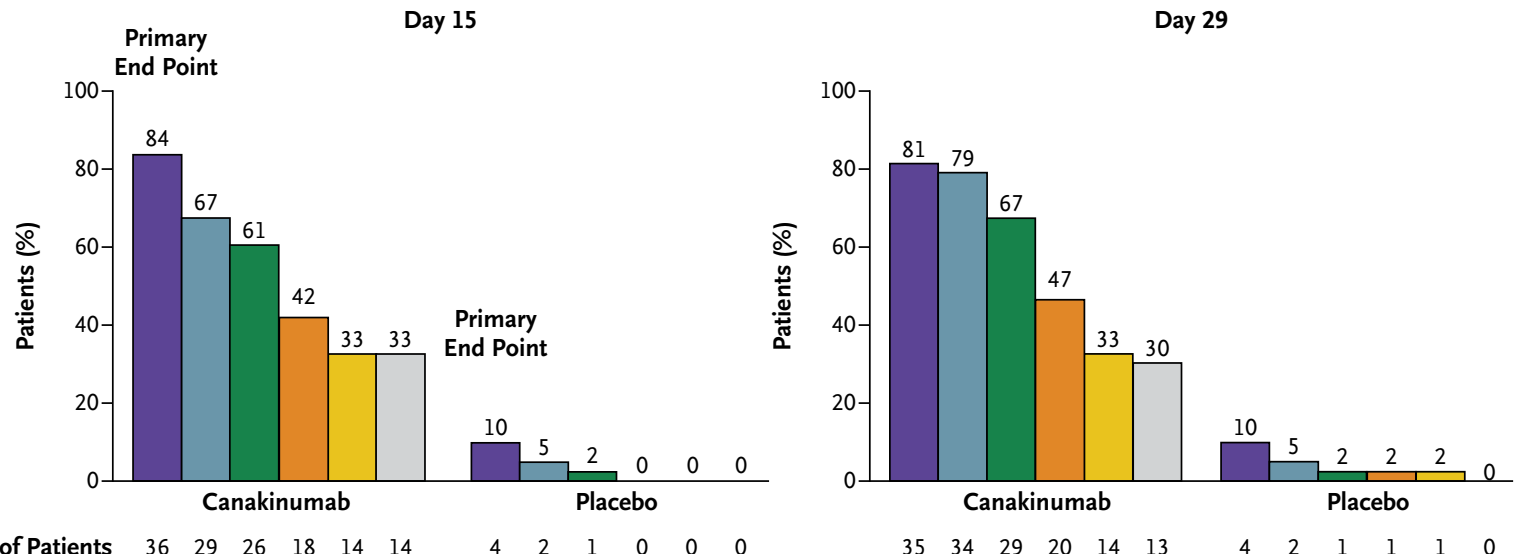

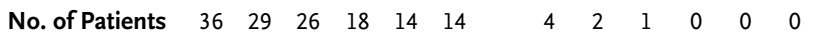

$\begin{array}{llllllllllll}35 & 34 & 29 & 20 & 14 & 13 & 4 & 2 & 1 & 1 & 1 & 0\end{array}$

B Trial 2

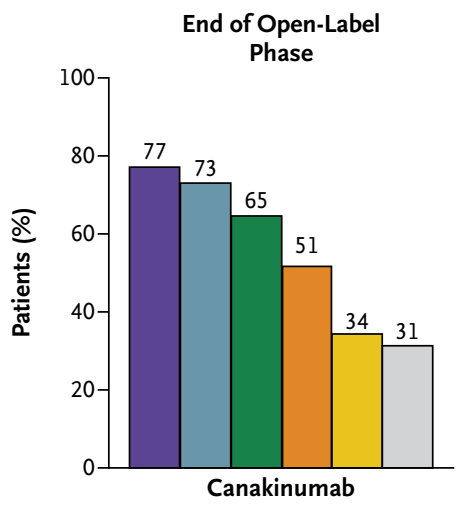

No. of Patients $\quad \begin{array}{llllll}135 & 128 & 113 & 90 & 60 & 55\end{array}$

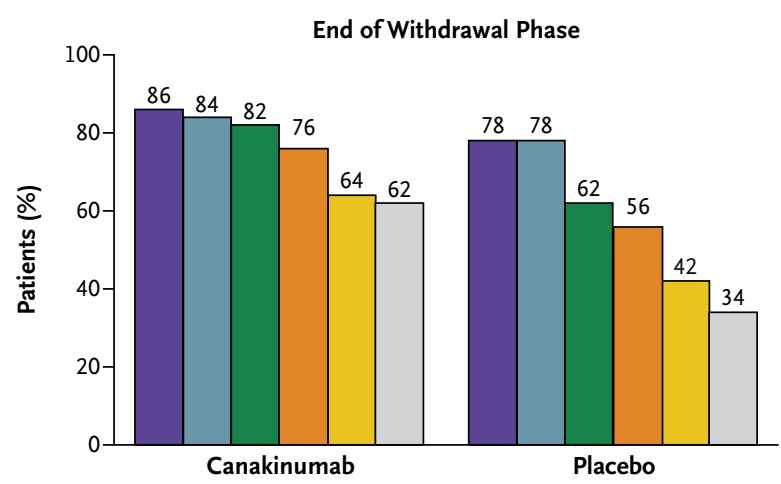

$\begin{array}{llllllllllll}43 & 42 & 41 & 38 & 32 & 31 & 39 & 39 & 31 & 28 & 21 & 17\end{array}$

Figure 1. Response to Treatment.

Panel A shows the rates of adapted American College of Rheumatology (ACR) responses for the core set of variables for systemic juvenile idiopathic arthritis (JIA) and inactive disease status in trial 1 (single dose of canakinumab vs. placebo). The adapted JIA ACR 30, JIA ACR 50, JIA ACR 70, JIA ACR 90, and JIA ACR 100 responses were defined as improvements of at least 30\%, 50\%, 70\%, 90\%, and $100 \%$, respectively, in at least three of the six response variables and a worsening of more than $30 \%$ in no more than one of the six variables, as well as absence of fever. $P<0.001$ for all comparisons of JIA ACR responses; $P$ values were not determined for comparisons regarding inactive disease. Panel B shows the rates of adapted JIA ACR responses and inactive disease status in trial 2 , both at the end of the openlabel phase and at the end of the withdrawal phase, which included data from the last observation of patients who had had a response. 


\begin{tabular}{|c|c|c|c|c|c|c|c|}
\hline \multirow[t]{3}{*}{ Variable } & \multicolumn{3}{|c|}{ Trial 1} & \multicolumn{4}{|c|}{ Trial 2} \\
\hline & \multicolumn{2}{|c|}{ Baseline } & \multirow{2}{*}{$\begin{array}{l}\text { End of Trial } \\
\text { Canakinumab } \\
\qquad(\mathrm{N}=38)\end{array}$} & \multirow{2}{*}{$\begin{array}{c}\text { Baseline } \\
\text { Canakinumab } \\
(\mathrm{N}=177)\end{array}$} & \multirow{2}{*}{$\begin{array}{l}\text { End of Open- } \\
\text { Label Phase } \\
\text { Canakinumab } \\
(\mathrm{N}=100)\end{array}$} & \multicolumn{2}{|c|}{ End of Withdrawal Phase } \\
\hline & $\begin{array}{l}\text { Placebo } \\
(\mathrm{N}=41)\end{array}$ & $\begin{array}{l}\text { Canakinumab } \\
\quad(\mathrm{N}=43)\end{array}$ & & & & $\begin{array}{l}\text { Canakinumab } \\
\quad(\mathrm{N}=50)\end{array}$ & $\begin{array}{l}\text { Placebo } \\
(N=50)\end{array}$ \\
\hline \multicolumn{8}{|c|}{ No. of joints with active arthritis $\uparrow$} \\
\hline Median & 7.0 & 10.0 & 1.0 & 10.0 & 0.0 & 0.0 & 0.0 \\
\hline Interquartile range & $4.0-19.0$ & $4.0-24.0$ & $0-6.0$ & $4.0-22.0$ & $0.0-2.0$ & $0.0-2.0$ & $0.0-4.0$ \\
\hline \multicolumn{8}{|c|}{$\begin{array}{l}\text { No. of joints with limited range } \\
\text { of motiont }\end{array}$} \\
\hline Median & 6.0 & 8.0 & 2.0 & 9.0 & 0.0 & 0.0 & 1.0 \\
\hline Interquartile range & $4.0-22.0$ & $4.0-19.0$ & $0.0-8.0$ & $4.0-23.0$ & $0.0-2.0$ & $0.0-2.0$ & $0.0-4.0$ \\
\hline \multicolumn{8}{|c|}{$\begin{array}{l}\text { Score for physician's global assessment } \\
\text { of disease activity } \mathbb{S}\end{array}$} \\
\hline Median & 66.0 & 67.0 & 11.0 & 70.0 & 0.0 & 0.0 & 6.5 \\
\hline Interquartile range & $55.0-80.0$ & $55.0-80.0$ & $1.0-29.0$ & $55.0-80.0$ & $0.0-6.0$ & $0.0-7.0$ & $0.0-30.0$ \\
\hline \multicolumn{8}{|c|}{$\begin{array}{l}\text { Score for parent's global assessment } \\
\text { of patient's overall well-being }\end{array}$} \\
\hline Median & 61.0 & 63.0 & 6.5 & 63.5 & 2.0 & 1.0 & 3.0 \\
\hline Interquartile range & $35.0-81.0$ & $45.0-81.0$ & $0.0-26.0$ & $45.0-80.0$ & $0.0-12.0$ & $0.0-7.0$ & $1.0-30.0$ \\
\hline \multicolumn{8}{|l|}{ CHAQ-DI score\| } \\
\hline Median & 1.5 & 1.6 & 0.2 & 1.8 & 0.0 & 0.0 & 0.1 \\
\hline Interquartile range & $1.0-2.1$ & $1.1-2.3$ & $0.0-0.9$ & $1.1-2.3$ & $0.0-0.4$ & $0.0-0.4$ & $0.0-1.0$ \\
\hline \multicolumn{8}{|l|}{ C-reactive protein** } \\
\hline Median & 137.0 & 141.3 & 12.0 & 160.0 & 5.3 & 5.0 & 17.9 \\
\hline Interquartile range & $71.2-194.9$ & $88.0-270.0$ & $3.3-76.6$ & $88.0-271.0$ & $1.8-16.5$ & $1.2-10.0$ & $3.3-68.0$ \\
\hline \multicolumn{8}{|c|}{ Systemic features — no./total no. (\%) †† } \\
\hline Fevertrt & $39 / 41(95)$ & $42 / 43(98)$ & $5 / 43(12)$ & $161 / 177(91)$ & $0 / 100$ & $3 / 50(6)$ & $9 / 50(18)$ \\
\hline Rash & $24 / 41(59)$ & $20 / 43(47)$ & $4 / 43(9)$ & 79/177 (45) & $1 / 100(1)$ & $3 / 50(6)$ & $9 / 50(18)$ \\
\hline
\end{tabular}

* Data are shown for patients who received canakinumab in trial 1 and trial 2. However, only baseline values are provided for patients who received placebo in trial 1 , since 37 of 41 patients in the placebo group $(90 \%)$ discontinued the study drug prematurely and were immediately enrolled in trial 2.

$\dagger$ The range of possible values for number of joints with active arthritis was 0 to $73 .{ }^{21}$

The range of possible values for number of joints with limited range of motion was 0 to 69 .

$\int$ The physician's global assessment of disease activity was based on a $100-\mathrm{mm}$ visual-analogue scale, with higher scores indicating more active disease.

9 The parent's global assessment of the patient's overall well-being was based on a $100-\mathrm{mm}$ visual-analogue scale, with higher scores indicating more active disease.

| Physical function was assessed by means of the cross-culturally adapted and validated version of the Childhood Health Assessment Questionnaire-Disability Index (CHAQ-DI), with scores ranging from 0 to 3 and higher scores indicating greater disability.

*** The level of C-reactive protein was standardized to the upper limit of the normal range of $10 \mathrm{mg}$ per liter (normal range, 0 to $10 \mathrm{mg}$ per liter). ${ }^{24,26-28}$

†† The total number of patients who were receiving canakinumab at the end of the trial is equal to the total number of patients who underwent randomization in trial 1 and who were treated with canakinumab. Some patients in trial 2 did not have fever or rash because these signs disappeared when they began participating in that trial.

$\rightarrow$ Resolution of fever was defined as the documented absence of fever due to systemic JIA (temperature, $\leq 38^{\circ} \mathrm{C}$ ) in the week preceding the assessment, according to documentation in a paper diary.

thrombocytopenia; 6 patients in the canakinumab group (12\%) and 1 in the placebo group (2\%) had neutropenia. One patient had both neutropenia and thrombocytopenia and another had throm- bocytopenia during the macrophage activation syndrome. Laboratory abnormalities resolved within a mean of 33 days in all patients for whom follow-up data were available. 


\begin{tabular}{|c|c|c|c|c|c|}
\hline \multirow[t]{2}{*}{ Event } & \multicolumn{2}{|c|}{ Trial 1} & \multirow{2}{*}{$\begin{array}{c}\text { Trial 2, } \\
\text { Open-Label Phase } \\
\text { Canakinumab } \\
(\mathrm{N}=177)\end{array}$} & \multicolumn{2}{|c|}{$\begin{array}{c}\text { Trial 2, } \\
\text { Withdrawal Phase }\end{array}$} \\
\hline & $\begin{array}{l}\text { Canakinumab } \\
\qquad(\mathrm{N}=43)\end{array}$ & $\begin{array}{l}\text { Placebo } \\
(\mathrm{N}=41)\end{array}$ & & $\begin{array}{l}\text { Canakinumab } \\
\qquad(\mathrm{N}=50)\end{array}$ & $\begin{array}{l}\text { Placebo } \\
(\mathrm{N}=50)\end{array}$ \\
\hline Total no. of adverse events & 49 & 27 & 664 & 272 & 229 \\
\hline Patients with event — no. (\%) & $24(56)$ & $16(39)$ & $138(78)$ & $40(80)$ & $35(70)$ \\
\hline Rate per 100 patient-days $†$ & NA & NA & NA & 2.34 & 2.53 \\
\hline Patients with serious adverse event — no. (\%) & $2(5)$ & $2(5)$ & $15(8)$ & $6(12)$ & $6(12)$ \\
\hline $\begin{array}{l}\text { Patients with adverse event leading to with- } \\
\text { drawal - no. (\%) }\end{array}$ & 0 & 0 & $5(3)$ & 0 & $6(12)$ \\
\hline \multicolumn{6}{|l|}{ Infection } \\
\hline Patients with event — no. (\%) & $13(30)$ & $5(12)$ & $97(55)$ & $27(54)$ & $19(38)$ \\
\hline Rate per 100 patient-days $\uparrow$ & NA & NA & NA & 0.59 & 0.63 \\
\hline Patients with serious infection - no. (\%) & $2(5)$ & $1(2)$ & $7(4)$ & $2(4)$ & $2(4)$ \\
\hline Macrophage activation syndrome - no. (\%) & $1(2)$ & $1(2)$ & $4(2)$ & 0 & $1(2)$ \\
\hline Death - no. (\%) & 0 & 0 & $1(1)$ & 0 & $1(2) \ddagger$ \\
\hline \multicolumn{6}{|l|}{ Most frequently reported events - no. (\%) $\mathbb{\int}$} \\
\hline Abdominal pain & $3(7)$ & $1(2)$ & $26(15)$ & $8(16)$ & $6(12)$ \\
\hline Cough & $1(2)$ & 0 & $20(11)$ & $8(16)$ & $6(12)$ \\
\hline Headache & $2(5)$ & $1(2)$ & $23(13)$ & $3(6)$ & $3(6)$ \\
\hline Nasopharyngitis & $3(7)$ & $1(2)$ & $27(15)$ & $7(14)$ & $7(14)$ \\
\hline Pyrexia & $2(5)$ & 0 & $18(10)$ & $7(14)$ & $5(10)$ \\
\hline Upper respiratory tract infection & $3(7)$ & 0 & $18(10)$ & $6(12)$ & $5(10)$ \\
\hline Vomiting & $1(2)$ & $1(2)$ & $18(10)$ & $1(2)$ & $4(8)$ \\
\hline
\end{tabular}

* The rates of adverse events and serious adverse events per patient-year were not calculated for trial 1 or for the openlabel phase of trial 2; these analyses were not planned because of the short duration of the trial or phase and the small number of patients in the placebo group. NA denotes not available.

$\dagger$ The frequency of adverse events in trial $l$ in the placebo and canakinumab groups must be interpreted with consideration of the median period of study participation, which was 8 days in the placebo group as compared with 29 days in the canakinumab group.

+ This patient died 7 days after transfer to a tertiary care hospital ( 2 days after withdrawal from the study).

$\int$ The most frequently reported events were defined as those that occurred in at least $10 \%$ of patients during the open-label phase of trial 2.

There were seven reported cases of the macrophage activation syndrome (including two cases in the placebo group [one during trial 1 and the other during the withdrawal phase of trial 2]). An independent adjudication committee classified five of the cases as probable cases of the macrophage activation syndrome and two as possible cases. With respect to the two possible cases, the committee stated that ferritin levels were low for the macrophage activation syndrome in one case, and in the other case, the rash was atypical and the patient was assessed as having a Klebsiella pneumoniae infection.

Two patients died while they were participating in trial 2. A 16-year-old girl had received eight doses of canakinumab, followed by placebo for 164 days (six doses); she then had progressive clinical worsening and urosepsis. Clinical, laboratory, and bone marrow findings suggested the macrophage activation syndrome. While being transferred to a tertiary care hospital, she had a cardiac arrest requiring resuscitation and intubation. The findings on chest radiography were consistent with the acute respiratory distress syndrome. The patient died 7 days after the transfer to the tertiary care hospital ( 2 days after withdrawal from the study). In addition, a 13-yearold boy, who had previously been treated with anakinra and tocilizumab, died during study participation. The child was hospitalized for an 
episode of adenovirus gastroenteritis, which resolved within 14 days. Four days later, after receiving the third dose of canakinumab, he was rehospitalized for the macrophage activation syndrome; severe pulmonary hypertension developed, and he died 3 weeks later.

In addition, two deaths occurred outside the studies. A 9-year-old girl died from disease progression approximately 3 months after trial discontinuation because of an unsatisfactory therapeutic effect. A 19-year-old woman died more than 2 years after receiving the last dose of canakinumab in the phase 2 trial $^{18}$; the patient's cerebrospinal-fluid and blood cultures were positive for Streptococcus pneumoniae.

\section{DISCUSSION}

We conducted two parallel studies to assess the efficacy and safety of canakinumab in the treatment of systemic JIA with active systemic features. The rationale for trial 1 was to provide evidence that the drug is effective in controlling fever within a few days after administration. For ethical reasons, patients in this trial who had persistent fever 3 days after the first injection were permitted, at the discretion of the physician, to be made aware of their study assignment in an effort to rapidly provide access to open-label treatment in trial 2. The rationale for trial 2 was to investigate the glucocorticoid-tapering potential and the prevention of disease flare during up to 2 years of treatment.

In trial 1, a single injection of canakinumab resulted in inactive disease in as few as 15 days in $33 \%$ of the patients, confirming the preliminary results of the phase 2 dose-finding study. ${ }^{18}$ The results of trial 1 were corroborated by those of trial 2, which showed that 31 (62\%) of 50 patients continuously treated with canakinumab had inactive disease status and $41(82 \%)$ had at least an adapted JIA ACR 70 response after 2 years of treatment. These findings confirm, in a controlled setting, previous observations with anakinra, ${ }^{11-13,18,35}$ which suggested that about $40 \%$ of patients with systemic JIA have a dramatic and persistent response to anti-interleukin- 1 therapy. This is similar to response rates observed in some autoinflammatory diseases ${ }^{15,17,35-37}$ and in trials with anti-interleukin-6 medications. ${ }^{8-10}$

In trial 2, the open-label phase lasted for 32 weeks to allow tapering of glucocorticoids to a safe dose, which then remained stable during the subsequent double-blind withdrawal phase. Canakinumab allowed for the discontinuation of glucocorticoids in $33 \%$ of the patients within 7 months after treatment, decreasing the burden of glucocorticoid toxicity among patients with a disease that often results in dependence on glucocorticoids. In the withdrawal phase, the median time to flare was 8 months in the placebo group (95\% CI, 5 to 15), whereas the median time to flare could not be estimated for the canakinumab group, owing to the low number of flares that had occurred at the time of study completion. ${ }^{18}$

For all treatment groups, the most common adverse events were infections. The crude incidence rate of infection during treatment with canakinumab was similar to that with placebo in these trials. Neutropenia and thrombocytopenia were observed in both trials and were mostly transient, isolated events that were not associated with an increased risk of infection or bleeding. Seven cases of the macrophage activation syndrome were reported in these trials, with two associated deaths. ${ }^{38,39}$ The mortality rate in phase $2^{18}$ and phase 3 trials of canakinumab (2\% over an average of 547 days) is in line with the mortality rate associated with systemic JIA, as reported by Hashkes et al. ${ }^{40}$

A limitation of the two studies is that patients without fever were excluded from participation. Indeed, in a subset of patients with systemic JIA, systemic symptoms eventually resolve while chronic arthritis continues. Therefore, the efficacy of canakinumab in patients who have systemic JIA without fever cannot be deduced directly from our results. In addition, information on the safety of canakinumab in patients with systemic JIA is limited, given the short duration of exposure to placebo in both trials and the use of a withdrawal design..$^{23,41,42}$ Longer-term safety data are needed.

In conclusion, these two placebo-controlled trials show the efficacy of canakinumab in the treatment of systemic JIA with active systemic features. Supported by Novartis Pharma.

Disclosure forms provided by the authors are available with the full text of this article at NEJM.org.

We thank the patients and their families, the staff from the Paediatric Rheumatology International Trials Organisation (PRINTO) and the Pediatric Rheumatology Collaborative Study Group (PRCSG) coordinating centers, all members of the PRINTO and PRCSG networks, and the Novartis team. 
The authors' affiliations are as follows: Istituto Giannina Gaslini, Pediatria II, Reumatologia, Paediatric Rheumatology International Trials Organisation (PRINTO) Coordinating Center (N.R., S.V., A.M.), and Dipartimento di Pediatria, Università di Genova, (A.M.), Genoa, and Azienda Ospedaliero-Universitaria Meyer, Florence (R.C.) - both in Italy; the Division of Rheumatology, Department of Pediatrics, Cincinnati Children's Hospital Medical Center, Pediatric Rheumatology Collaborative Study Group (PRCSG) Coordinating Center, Cincinnati (H.I.B., A.G., D.J.L.); Unité d'Immunologie, Hématologie et Rhumatologie Pediatrique, Université Paris-Descartes, Hôpital Necker-Enfants Malades (P.Q.), and Centre de Reference National des Maladies Auto-Inflammatoires, Rhumatologie Pediatrique, Centre Hospitalier Universitaire le Kremlin-Bicêtre, University of Paris Sud, Le Kremlin-Bicêtre (I.K.-P) — both in France; Unit of Pediatric Rheumatology, Semmelweis University, Budapest, Hungary (T.C.); Department of Pediatric Immunology and Rheumatology, Wilhelmina Kinderziekenhuis, Utrecht, the Netherlands (N.W.); Zentrum für Allgemeine Paediatrie und Neonatologie, Sankt Augustin (G.H.), and Pediatric Pneumology and Immunology, Charité University Hospital Berlin, Berlin (T.K.) — both in Germany; Department of Pediatrics, Rambam Medical Center, Haifa (R.B.), Department of Pediatrics, Hadassah Medical Center-Hadassah University Hospital Mount Scopus, Jerusalem (Y.B.), and the Department of Pediatrics, Meir Medical Center, Kfar Saba (Y.U.) - all in Israel; Alder Hey Children's National Health Service Foundation Trust, Liverpool (L.M.), and Centre of Paediatric and Adolescent Rheumatology, Great Ormond Street Hospital, University College London, London (K.N.) - both in the United Kingdom; Pediatric Rheumatology, Cerrahpasa Medical Faculty, Istanbul University (O.K.), and Clinic of Pediatric Health and Diseases, Göztepe Education and Research Hospital (M.E.), Istanbul, and the Department of Pediatric Nephrology and Rheumatology, Hacettepe University Children's Hospital, Ankara (S.O.) — all in Turkey; Pediatric Clinic, Institute of Rheumatology, Warsaw, Poland (L.R.-S.); Division of Rheumatology, Hospital for Sick Children, University of Toronto, Toronto (R.S.); Pediatric Rheumatology Department, Hospital Universitario La Fe, Valencia (I.C.), and Unidad de Reumatología Pediátrica, Hospital Ramon y Cajal, Madrid (M.L.G.) — both in Spain; Service de Pediatrie, Hôpital Universitaire des Enfants Reine Fabiola, Université Libre de Bruxelles, Brussels (L.G.), and the Department of Pediatrics, Division of Pediatric Rheumatology, University Hospital Gasthuisberg, Leuven (C.W.) — both in Belgium; Pediatrie, Unité Romande de Rhumatologie Pediatrique, Centre Hospitalier Universitaire Vaudois, University of Lausanne, Lausanne, and Hôpitaux Universitaires de Genève, Geneva (M.H.), and Novartis Pharma, Basel (K.L.) — all in Switzerland; Instituto de Puericultura e Pediatria Martagao Gesteira, Universidade Federal do Rio de Janeiro (S.K.O.), and Nucleo de Estudos da Saúde do Adolescente, Hospital Universitario Pedro Ernesto (F.S.) - both in Rio de Janeiro; Instituto Nacional de Salud del Niño, Breña, Lima, Peru (M.A.F.); Department of Rheumatology, Rikshospitalet University Hospital, Oslo (B.F.); Pediatric Rheumatology Unit, Karolinska University Hospital, Stockholm (B.M.); and Novartis Pharmaceuticals, East Hanover, NJ (K.A., D.K.).

REFERENCES

1. Prakken B, Albani S, Martini A. Juvenile idiopathic arthritis. Lancet 2011;377: 2138-49.

2. Ravelli A, Martini A. Juvenile idiopathic arthritis. Lancet 2007;369:767-78.

3. Martini A, Ravelli A, Di Fuccia G, Rosti V, Cazzola M, Barosi G. Intravenous iron therapy for severe anaemia in systemic-onset juvenile chronic arthritis. Lancet 1994;344:1052-4.

4. Packham JC, Hall MA. Long-term follow-up of 246 adults with juvenile idiopathic arthritis: functional outcome. Rheumatology (Oxford) 2002;41:1428-35. 5. De Benedetti F, Alonzi T, Moretta A, et al. Interleukin 6 causes growth impairment in transgenic mice through a decrease in insulin-like growth factor-I: a model for stunted growth in children with chronic inflammation. J Clin Invest 1997;99:643-50.

6. Ravelli A, Magni-Manzoni S, Pistorio A, et al. Preliminary diagnostic guidelines for macrophage activation syndrome complicating systemic juvenile idiopathic arthritis. J Pediatr 2005;146:598-604.

7. Cassidy JT, Petty RE, Laxer RM, Lindsley CB. Textbook of pediatric rheumatology. 6th ed. Philadelphia: Saunders, 2010. 8. De Benedetti F, Martini A. Is systemic juvenile rheumatoid arthritis an interleukin 6 mediated disease? J Rheumatol 1998;25:203-7.

9. Yokota S, Imagawa $\mathrm{T}$, Mori $\mathrm{M}$, et al. Efficacy and safety of tocilizumab in patients with systemic-onset juvenile idiopathic arthritis: a randomised, double- blind, placebo-controlled, withdrawal phase III trial. Lancet 2008;371:998-1006. 10. De Benedetti F, Brunner HI, Ruperto N, et al. Randomized trial of tocilizumab in systemic juvenile idiopathic arthritis. N Engl J Med 2012;367:2385-95.

11. Pascual V, Allantaz F, Arce E, Punaro $\mathrm{M}$, Banchereau J. Role of interleukin-1 (IL-1) in the pathogenesis of systemic onset juvenile idiopathic arthritis and clinical response to IL-1 blockade. J Exp Med 2005;201:1479-86.

12. Gattorno M, Piccini A, Lasiglie D, et al. The pattern of response to anti-interleukin-1 treatment distinguishes two subsets of patients with systemic-onset juvenile idiopathic arthritis. Arthritis Rheum 2008;58:1505-15.

13. Quartier P, Allantaz F, Cimaz R, et al. A multicentre, randomised, double-blind, placebo-controlled trial with the interleukin-1 receptor antagonist anakinra in patients with systemic-onset juvenile idiopathic arthritis (ANAJIS trial). Ann Rheum Dis 2011;70:747-54.

14. Nigrovic PA, Mannion M, Prince FH, et al. Anakinra as first-line disease-modifying therapy in systemic juvenile idiopathic arthritis: report of forty-six patients from an international multicenter series. Arthritis Rheum 2011;63:545-55.

15. Lachmann HJ, Kone-Paut I, KuemmerleDeschner JB, et al. Use of canakinumab in the cryopyrin-associated periodic syndrome. N Engl J Med 2009;360:2416-25. 16. Kuemmerle-Deschner JB, Ramos E, Blank N, et al. Canakinumab (ACZ885, a fully human IgG1 anti-IL-1beta mAb) induces sustained remission in pediatric patients with cryopyrin-associated periodic syndrome (CAPS). Arthritis Res Ther 2011;13(1):R34.

17. Gattorno M, Pelagatti MA, Meini A, et al. Persistent efficacy of anakinra in patients with tumor necrosis factor receptor-associated periodic syndrome. Arthritis Rheum 2008;58:1516-20.

18. Ruperto N, Quartier P, Wulffraat N, et al. A phase II, multicenter, open-label study evaluating dosing and preliminary safety and efficacy of canakinumab in systemic juvenile idiopathic arthritis with active systemic features. Arthritis Rheum 2012;64:557-67.

19. Petty RE, Southwood TR, Manners P, et al. International League of Associations for Rheumatology classification of juvenile idiopathic arthritis: second revision, Edmonton, 2001. J Rheumatol 2004; 31:390-2.

20. Petty RE, Southwood TR, Baum J, et al. Revision of the proposed classification criteria for juvenile idiopathic arthritides: Durban, 1997. J Rheumatol 1998;25:1991-4. 21. Cassidy JT, Levinson JE, Bass JC, et al. A study of classification criteria for a diagnosis of juvenile rheumatoid arthritis. Arthritis Rheum 1986;29:274-81.

22. Ruperto $\mathrm{N}$, Martini A. Networking in pediatrics: the example of the Pediatric Rheumatology International Trials Organisation (PRINTO). Arch Dis Child 2011;96: 596-601.

23. Ruperto N, Giannini EH, Pistorio A, 
Brunner HI, Martini A, Lovell DJ. Is it time to move to active comparator trials in juvenile idiopathic arthritis? A review of current study designs. Arthritis Rheum 2010;62:3131-9.

24. Giannini EH, Ruperto N, Ravelli A, Lovell DJ, Felson DT, Martini A. Preliminary definition of improvement in juvenile arthritis. Arthritis Rheum 1997;40: 1202-9.

25. Giannini EH, Lovell DJ, Silverman ED, Sundel RP, Tague BL, Ruperto N. Intravenous immunoglobulin in the treatment of polyarticular juvenile rheumatoid arthritis: a phase I/II study. J Rheumatol 1996; 23:919-24.

26. Singh G, Athreya BH, Fries JF, Goldsmith DP. Measurement of health status in children with juvenile rheumatoid arthritis. Arthritis Rheum 1994;37:1761-9.

27. Paediatric Rheumatology International Trials Organisation (PRINTO). Quality of life in juvenile idiopathic arthritis patients compared to healthy children. Clin Exp Rheumatol 2001;19:Suppl 23:S1-172.

28. Ruperto N, Ravelli A, Pistorio A, et al. Cross-cultural adaptation and psychometric evaluation of the Childhood Health Assessment Questionnaire (CHAQ) and the Child Health Questionnaire (CHQ) in 32 countries: review of the general methodology. Clin Exp Rheumatol 2001;19:S1S9.

29. Brunner HI, Lovell DJ, Finck BK, Gi- annini EH. Preliminary definition of disease flare in juvenile rheumatoid arthritis. J Rheumatol 2002;29:1058-64.

30. Wallace CA, Ruperto N, Giannini E. Preliminary criteria for clinical remission for select categories of juvenile idiopathic arthritis. J Rheumatol 2004;31:2290-4.

31. Wallace CA, Giannini EH, Huang B, Itert L, Ruperto N. American College of Rheumatology provisional criteria for defining clinical inactive disease in select categories of juvenile idiopathic arthritis Arthritis Care Res (Hoboken) 2011;63:929 36.

32. Griffin TA, Barnes MG, Ilowite NT, et al. Gene expression signatures in polyarticular juvenile idiopathic arthritis demonstrate disease heterogeneity and offer a molecular classification of disease subsets. Arthritis Rheum 2009;60:211323.

33. Ruperto N, Lovell DJ, Quartier P, et al Abatacept in children with juvenile idiopathic arthritis: a randomised, doubleblind, placebo-controlled withdrawal trial. Lancet 2008;372:383-91.

34. Schulz KF, Altman DG, Moher D, Fergusson D. CONSORT 2010 changes and testing blindness in RCTs. Lancet 2010 375:1144-6

35. Lachmann HJ, Quartier P, So A Hawkins PN. The emerging role of interleukin- $\beta$ in autoinflammatory diseases. Arthritis Rheum 2011;63:314-24.
36. Picco P, Brisca G, Traverso F, Loy A, Gattorno M, Martini A. Successful treatment of idiopathic recurrent pericarditis in children with interleukin-1beta receptor antagonist (anakinra): an unrecognized autoinflammatory disease? Arthritis Rheum 2009;60:264-8.

37. Lepore L, Paloni G, Caorsi R, et al. Follow-up and quality of life of patients with cryopyrin-associated periodic syndromes treated with anakinra. J Pediatr 2010;157:310-5.

38. Padeh S, Laxer RM, Silver MM, Silverman ED. Primary pulmonary hypertension in a patient with systemic-onset juvenile arthritis. Arthritis Rheum 1991;34:1575-9. 39. Kimura Y, Weiss JE, Haroldson KL, et al. Pulmonary hypertension and other potentially fatal pulmonary complications in systemic juvenile idiopathic arthritis. Arthritis Care Res (Hoboken) 2012 November 8 (Epub ahead of print).

40. Hashkes PJ, Wright BM, Lauer MS, et al. Mortality outcomes in pediatric rheumatology in the US. Arthritis Rheum 2010; 62:599-608.

41. Sfriso P, Ravaioli F. Adalimumab in juvenile rheumatoid arthritis. N Engl J Med 2008;359:2495-7.

42. De Vries MK, Van der Horst-Bruinsma IE, Wolbink GJ. Adalimumab in juvenile rheumatoid arthritis. N Engl J Med 2008; 359:2496-7.

Copyright (c) 2012 Massachusetts Medical Society. 